Georgian Mathematical Journal

Volume 13 (2006), Number 1, 85-99

\title{
ON GENERATING RELATIONS INVOLVING GENERALIZED GEGENBAUER POLYNOMIALS
}

\author{
SUBUHI KHAN, GHAZALA YASMIN, AND ARCHANA MITTAL
}

\begin{abstract}
In this paper, generating relations involving generalized Gegenbauer polynomials $C_{n}^{(\mu)}(x, y ; \alpha)$ are obtained by constructing a three-dimensional Lie algebra isomorphic to special linear algebra sl(2). Further, a number of new interesting relations involving various generalized polynomials are obtained as applications of these generating relations.
\end{abstract}

2000 Mathematics Subject Classification: 33C50, 33C80, 33C05.

Key words and phrases: Generalized Gegenbauer polynomials, Lie group, Lie algebra, generating relations.

\section{INTRODUCTION}

Chebyshev polynomials of second kind are defined by [4]

$$
U_{n}(x)=\sum_{k=0}^{[n / 2]} \frac{(-1)^{k}(n-k) !(2 x)^{n-2 k}}{k !(n-2 k) !},
$$

and the generating function for these polynomials is

$$
\left(1-2 x t+t^{2}\right)^{-1}=\sum_{n=0}^{\infty} U_{n}(x) t^{n} .
$$

We note the following integral representation for these polynomials ([3]; p. $418(7))$

$$
U_{n}(x)=\frac{1}{n !} \int_{0}^{\infty} e^{-t} t^{n} H_{n}\left(2 x,-\frac{1}{t}\right) d t
$$

where $H_{n}(x, y)$ are two variable Hermite polynomials (2VHP) defined by the series [3]

$$
H_{n}(x, y)=n ! \sum_{k=0}^{[n / 2]} \frac{x^{n-2 k} y^{k}}{k !(n-2 k) !} .
$$

Chebyshev polynomials are particular cases of the Gegenbauer polynomials defined by [13]

$$
C_{n}^{(\mu)}(x)=\frac{1}{\Gamma(\mu)} \sum_{k=0}^{[n / 2]} \frac{(-1)^{k} \Gamma(n+\mu-k)(2 x)^{n-2 k}}{k !(n-2 k) !}
$$


with the generating function

$$
\left(1-2 x t+t^{2}\right)^{-\mu}=\sum_{n=0}^{\infty} C_{n}^{(\mu)}(x) t^{n} .
$$

Dattoli et al. [1] used the method of integral transform to generalize Gegenbauer polynomials.

We consider the two-variable one-parameter Gegenbauer polynomials (2V1PGP) defined by ([1]; p. 11(23))

$$
C_{n}^{(\mu)}(x, y ; \alpha)=\frac{1}{\Gamma(\mu)} \sum_{k=0}^{[n / 2]} \frac{\Gamma(n+\mu-k)(2 x)^{n-2 k}(-y)^{k}}{k !(n-2 k) ! \alpha^{n+\mu-k}} .
$$

The generating function for these polynomials is

$$
\left(\alpha-2 x t+y t^{2}\right)^{-\mu}=\sum_{n=0}^{\infty} C_{n}^{(\mu)}(x, y ; \alpha) t^{n}
$$

and the integral representation for these polynomials in terms of $2 \mathrm{VHP} H_{n}(x, y)$ is

$$
C_{n}^{(\mu)}(x, y ; \alpha)=\frac{1}{n ! \Gamma(\mu)} \int_{0}^{\infty} e^{-\alpha t} t^{n+\mu-1} H_{n}\left(2 x,-\frac{y}{t}\right) d t
$$

Motivated and inspired by the works of Dattoli et al. [1]-[3] and a recent work on the representation of a Lie algebra $\mathcal{G}(0,1)$ and two-dimensional Hermite polynomials [8], we derive generating relations involving $2 \mathrm{~V} 1 \mathrm{PGP} C_{n}^{(\mu)}(x, y ; \alpha)$ and hypergeometric function ${ }_{2} F_{1}[13]$ by constructing a three-dimensional Lie algebra isomorphic to special linear algebra $\mathrm{sl}(2)$, the Lie algebra of the complex special linear group $S L(2)$ ([10]; p. 7), using Weisner's method [15].

The reason for our interest in this family of polynomials is due to the fact that these polynomials include as their special cases various other generalized as well as known polynomials, which we discuss in Section 2. The Group-theoretic method is discussed in Section 3. Using the representation of the Lie group $S L(2)$, generating relations are derived in Section 4. The main interest in our results lies in the fact that a number of special cases can be used to derive many new and known results for the polynomials associated with 2V1PGP, which are obtained in Section 5. Finally, some concluding remarks are given in Section 6 .

\section{Properties of 2V1PGP $C_{n}^{(\mu)}(x, y ; \alpha)$}

The 2V1PGP $C_{n}^{(\mu)}(x, y ; \alpha)$ satisfy the differential equation

$$
\left(\left(x^{2}-\alpha y\right) \frac{d^{2}}{d x^{2}}+(1+2 \mu) x \frac{d}{d x}-n(n+2 \mu)\right) C_{n}^{(\mu)}(x, y ; \alpha)=0 .
$$


The differential and pure recurrence relations satisfied by these polynomials are

$$
\begin{gathered}
\frac{\partial}{\partial x} C_{n}^{(\mu)}(x, y ; \alpha)=\frac{1}{x}\left(n C_{n}^{(\mu)}(x, y ; \alpha)-y \frac{\partial}{\partial x} C_{n-1}^{(\mu)}(x, y ; \alpha)\right) \\
\frac{\partial}{\partial x} C_{n}^{(\mu)}(x, y ; \alpha)=\frac{1}{\left(x^{2}-\alpha y\right)}\left(\alpha(n+1) C_{n+1}^{(\mu)}(x, y ; \alpha)\right. \\
\left.\quad-(n+2 \mu) x C_{n}^{(\mu)}(x, y ; \alpha)\right) \\
\frac{\partial}{\partial x} C_{n}^{(\mu)}(x, y ; \alpha)=\frac{1}{\left(x^{2}-\alpha y\right)}\left(n x C_{n}^{(\mu)}(x, y ; \alpha)\right. \\
\left.\quad-(n+2 \mu-1) y C_{n-1}^{(\mu)}(x, y ; \alpha)\right), \\
\begin{aligned}
\alpha(n+1) C_{n+1}^{(\mu)}(x, y ; \alpha)+(n+2 \mu-1) y C_{n-1}^{(\mu)}(x, y ; \alpha) \\
=2 x(n+\mu) C_{n}^{(\mu)}(x, y ; \alpha) .
\end{aligned}
\end{gathered}
$$

We note the following special cases of 2V1PGP $C_{n}^{(\mu)}(x, y ; \alpha)$ :

1.

$$
C_{n}^{(1)}(x, y ; \alpha)=U_{n}(x, y ; \alpha)
$$

where $U_{n}(x, y ; \alpha)$ denotes the two variable one-parameter Chebyshev polynomials (2V1PCP) defined by the generating function [1]

$$
\left(\alpha-2 x t+y t^{2}\right)^{-1}=\sum_{n=0}^{\infty} U_{n}(x, y ; \alpha) t^{n} .
$$

2.

$$
C_{n}^{(1 / 2)}(x, y ; \alpha)=P_{n}(x, y ; \alpha),
$$

where $P_{n}(x, y ; \alpha)$ denotes two variable one-parameter Legendre polynomials (2V1PLeP) defined by the generating function [1]

$$
\left(\alpha-2 x t+y t^{2}\right)^{-1 / 2}=\sum_{n=0}^{\infty} P_{n}(x, y ; \alpha) t^{n}
$$

3.

$$
C_{n}^{(\mu)}(x, y ; \alpha)=P_{n}(2, x, y,-\mu, \alpha),
$$

where $P_{n}(2, x, y,-\mu, \alpha)$ denotes generalized Humbert polynomials (GHP) defined by the generating function ([7]; p. 697(1.1))

$$
\left(c-m x t+y t^{m}\right)^{p}=\sum_{n=0}^{\infty} P_{n}(m, x, y, p, c) t^{n} .
$$

4.

$$
C_{n}^{(\mu)}(x, 0 ; \alpha)=C_{n}^{(\mu)}(x ; \alpha)=\frac{(\mu)_{n}(2 x)^{n}}{n ! \alpha^{n+\mu}},
$$

where the Pochhammer symbol $(\mu)_{n}$ is defined by

$$
(\mu)_{n}=\left\{\begin{aligned}
1, & \text { if } n=0 \\
\mu(\mu+1)(\mu+2) \cdots(\mu+n-1), & \text { if } n=1,2,3 \ldots
\end{aligned}\right.
$$

5.

$$
U_{n}(x, 0 ; \alpha)=U_{n}(x ; \alpha)=\frac{(2 x)^{n}}{\alpha^{n+1}} .
$$


6.

$$
C_{n}^{(\mu)}(x, 1 ; 1)=C_{n}^{(\mu)}(x),
$$

where $C_{n}^{(\mu)}(x)$ are the Gagenbauer polynomials (GP) defined by equation (1.6).

$$
\text { 7. } \quad C_{n}^{(1)}(x, 1 ; 1)=U_{n}(x),
$$

where $U_{n}(x)$ are the Chebyshev polynomials (CP) defined by equation (1.2).

8.

$$
C_{n}^{(1 / 2)}(x, 1 ; 1)=P_{n}(x),
$$

where $P_{n}(x)$ are the Legendre polynomials (LeP) defined by the generating function [13]

$$
\left(1-2 x t+t^{2}\right)^{-1 / 2}=\sum_{n=0}^{\infty} P_{n}(x) t^{n} .
$$

In the next section, to make use of the Lie group-theoretic method, we construct the partial differential equation corresponding to the differential equation (2.1) for $C_{n}^{(\mu)}(x, y ; \alpha)$. Further, we use the recurrence relations (2.2) to obtain first order linear differential operators which form the basis of defining the transformed function and the multiplier representation of the Lie group $S L(2)$.

\section{Group-Theoretic Discussion}

In order to use the Lie group-theoretic method, we replace $\frac{d}{d x}$ by $\frac{\partial}{\partial x}, n$ by $t \frac{\partial}{\partial t}$ and $C_{n}^{(\mu)}(x, y ; \alpha)$ by $f(x, y, t ; \alpha)$ in equation $(2.1)$ to construct the partial differential equation

$$
\left(\left(x^{2}-\alpha y\right) \frac{\partial^{2}}{\partial x^{2}}-t^{2} \frac{\partial^{2}}{\partial t^{2}}+(1+2 \mu) x \frac{\partial}{\partial x}-(1+2 \mu) t \frac{\partial}{\partial t}\right) f(x, y, t ; \alpha)=0 .
$$

Let $L$ represent the differential operator of equation (3.1), i.e.,

$$
L=L\left(x, \frac{\partial}{\partial x}, t \frac{\partial}{\partial t}\right)=\left(x^{2}-\alpha y\right) \frac{\partial^{2}}{\partial x^{2}}-t^{2} \frac{\partial^{2}}{\partial t^{2}}+(1+2 \mu) x \frac{\partial}{\partial x}-(1+2 \mu) t \frac{\partial}{\partial t} .
$$

Therefore $f(x, y, t ; \alpha)=C_{n}^{(\mu)}(x, y ; \alpha) t^{n}$ is a solution of equation (3.1), since $C_{n}^{(\mu)}(x, y ; \alpha)$ is a solution of equation (2.1).

We now seek for linearly independent maintaining, lowering and raising operators $J^{3}, J^{-}$and $J^{+}$, each of the form

$$
A_{1}(x, y, t) \frac{\partial}{\partial x}+A_{2}(x, y, t) \frac{\partial}{\partial y}+A_{3}(x, y, t) \frac{\partial}{\partial t}+A_{4}(x, y, t),
$$

defined on $\mathcal{F}$, the complex space of all functions analytic in some neighbourhood of $\left(x^{0}, y^{0}, t^{0}\right) \in \mathbb{C}^{3}$, such that

$$
\begin{aligned}
& J^{3}\left[C_{n}^{(\mu)}(x, y ; \alpha) t^{n}\right]=a_{n} C_{n}^{(\mu)}(x, y ; \alpha) t^{n}, \\
& J^{-}\left[C_{n}^{(\mu)}(x, y ; \alpha) t^{n}\right]=b_{n} C_{n-1}^{(\mu)}(x, y ; \alpha) t^{n-1}, \\
& J^{+}\left[C_{n}^{(\mu)}(x, y ; \alpha) t^{n}\right]=c_{n} C_{n+1}^{(\mu)}(x, y ; \alpha) t^{n+1},
\end{aligned}
$$

where $a_{n}, b_{n}$ and $c_{n}$ are expressions in $n$ which are independent of $x, y$ and $t$ but not necessarily of $\alpha$ and $\mu$. Each $A_{i}(x, y, t),(i=1,2,3,4)$, on the other hand, 
is an expression in $x, y$ and $t$ which is independent of $n$ but not necessarily of $\alpha$ and $\mu$.

Now using equation (3.3) and the recurrence relations (2.2), we get the operators

$$
\begin{aligned}
J^{3} & =t \frac{\partial}{\partial t}+\mu, \\
J^{-} & =\left(\frac{x^{2}-\alpha y}{y t}\right) \frac{\partial}{\partial x}-\frac{x}{y} \frac{\partial}{\partial t}, \\
J^{+} & =\left(\frac{x^{2}-\alpha y}{\alpha}\right) t \frac{\partial}{\partial x}+\frac{x t^{2}}{\alpha} \frac{\partial}{\partial t}+\frac{2 \mu x t}{\alpha} .
\end{aligned}
$$

Theorem 3.1. Three linearly independent linear operators $J^{3}, J^{-}$and $J^{+}$ given by equation (3.4) defined on $\mathcal{F}$ generate a three-dimensional Lie algebra isomorphic to $\mathrm{sl}(2)$, the Lie algebra of the complex special linear group $S L(2)$.

Proof. We observe that the operators $J^{3}, J^{-}$and $J^{+}$satisfy the following commutation relations

$$
\left[J^{3}, J^{ \pm}\right]= \pm J^{ \pm}, \quad\left[J^{+}, J^{-}\right]=2 J^{3}
$$

These commutation relations are identical with the commutation relations satisfied by the basis elements of the special linear algebra sl(2), the Lie algebra of the complex special linear group $S L(2)$, which is the abstract matrix group of all $2 \times 2$ nonsingular matrices

$$
g=\left(\begin{array}{ll}
a & b \\
c & d
\end{array}\right), \quad a, b, c, d \in \mathbb{C},
$$

such that $\operatorname{det} g=1$. The basis elements for $\operatorname{sl}(2)$ are provided by the matrices

$$
\mathcal{J}^{+}=\left(\begin{array}{cc}
0 & -1 \\
0 & 0
\end{array}\right), \quad \mathcal{J}^{-}=\left(\begin{array}{cc}
0 & 0 \\
0 & -1
\end{array}\right), \quad \mathcal{J}^{3}=\left(\begin{array}{cc}
1 / 2 & 0 \\
0 & -1 / 2
\end{array}\right) .
$$

Thus we conclude that the $J$-operators $J^{3}, J^{-}$and $J^{+}$generate a threedimensional Lie algebra isomorphic to $\mathrm{sl}(2)$.

In terms of the $J$-operators, we introduce the Casimir operator ([10]; p. 32),

$$
\begin{aligned}
\mathcal{C}=J^{+} J^{-}+J^{3} J^{3}-J^{3}= & \frac{\left(x^{2}-\alpha y\right)}{\alpha y}\left(\left(x^{2}-\alpha y\right) \frac{\partial^{2}}{\partial x^{2}}-t^{2} \frac{\partial^{2}}{\partial t^{2}}\right. \\
& \left.+(1+2 \mu) x \frac{\partial}{\partial x}-(1+2 \mu) t \frac{\partial}{\partial t}\right)+\mu(\mu-1) .
\end{aligned}
$$

It is easy to verify that the $J$-operators commute with the Casimir operator $\mathcal{C}$, that is

$$
\left[\mathcal{C}, J^{3}\right]=\left[\mathcal{C}, J^{ \pm}\right]=0
$$

Equation (3.8) enables us to rewrite equation (3.1) as

$$
\mathcal{C} f(x, y, t ; \alpha)=\mu(\mu-1) f(x, y, t ; \alpha) .
$$


Extended forms of the transformation groups generated by the operators $J^{3}, J^{-}$and $J^{+}$can be expressed as follows:

$$
\begin{aligned}
& e^{\tau^{\prime} J^{3}} f(x, y, t ; \alpha)=e^{\tau^{\prime} \mu} f\left(x, y, t e^{\tau^{\prime}} ; \alpha\right), \\
& e^{c^{\prime} J^{-}} f(x, y, t ; \alpha)=f\left(\frac{\xi \sqrt{\alpha y}}{\sqrt{\xi^{2}-t^{2} \theta}}, y, \frac{1}{\sqrt{\alpha y}} \sqrt{\xi^{2}-t^{2} \theta} ; \alpha\right), \\
& e^{b^{\prime} J^{+}} f(x, y, t ; \alpha)=\left(\frac{\alpha y}{\phi^{2}-\theta}\right)^{\mu} f\left(\frac{\phi \sqrt{\alpha y}}{\sqrt{\phi^{2}-\theta}}, y, \frac{t \sqrt{\alpha y}}{\sqrt{\phi^{2}-\theta}} ; \alpha\right),
\end{aligned}
$$

where $b^{\prime}, c^{\prime}$ and $\tau^{\prime}$ are arbitrary constants; $\xi:=\left(x t-\alpha c^{\prime}\right) ; \theta:=\left(x^{2}-\alpha y\right)$; $\phi:=\left(x-b^{\prime} y t\right)$ and $f(x, y, t ; \alpha)$ is an arbitrary function.

Thus it is obvious that

$$
\begin{aligned}
e^{b^{\prime} J^{+}} e^{c^{\prime} J^{-}}\left[C_{n}^{(\mu)}(x, y ; \alpha) t^{n}\right] \\
=(\alpha y)^{\mu-n / 2}\left(\phi^{2}-\theta\right)^{-(\mu+n / 2)}\left(\alpha^{2} y^{2} t^{2}-2 \alpha^{2} c^{\prime} y t \phi+\alpha^{2} c^{\prime 2}\left(\phi^{2}-\theta\right)\right)^{n / 2} \\
\quad \times C_{n}^{(\mu)}\left(\frac{\sqrt{\alpha y}\left(\alpha y t \phi-\alpha c^{\prime}\left(\phi^{2}-\theta\right)\right)}{\sqrt{\left(\phi^{2}-\theta\right)\left(\alpha^{2} y^{2} t^{2}-2 \alpha^{2} c^{\prime} y t \phi+\alpha^{2} c^{\prime 2}\left(\phi^{2}-\theta\right)\right)}}, y ; \alpha\right) .
\end{aligned}
$$

Now we proceed to compute the multiplier representation $[T(g) f](x, y, t ; \alpha)$, $g \in S L(2)$, induced by the $J$-operators.

For $g \in S L(2)$ and $d \neq 0$, by straightforward computation we show that

$$
g=\exp \left(b^{\prime} \mathcal{J}^{+}\right) \exp \left(c^{\prime} \mathcal{J}^{-}\right) \exp \left(\tau^{\prime} \mathcal{J}^{3}\right)
$$

where $b^{\prime}=-\frac{b}{d}, c^{\prime}=-c d$, $\exp \left(\frac{\tau^{\prime}}{2}\right)=\frac{1}{d}$ and $a d-b c=1$.

Hence the operator $T(g)$ is given by

$$
\begin{gathered}
{[T(g) f](x, y, t ; \alpha)=\left[T\left(\exp \left(b^{\prime} \mathcal{J}^{+}\right)\right) T\left(\exp \left(c^{\prime} \mathcal{J}^{-}\right)\right) T\left(\exp \left(\tau^{\prime} \mathcal{J}^{3}\right)\right) f\right](x, y, t ; \alpha)} \\
=e^{\mu \tau^{\prime}}\left(\frac{\alpha y}{\phi^{2}-\theta}\right)^{\mu} f\left(\frac{\sqrt{\alpha y}\left(\alpha y t \phi-\alpha c^{\prime}\left(\phi^{2}-\theta\right)\right)}{\sqrt{\left(\phi^{2}-\theta\right)\left(\alpha^{2} y^{2} t^{2}-2 \alpha^{2} c^{\prime} y t \phi+\alpha^{2} c^{\prime 2}\left(\phi^{2}-\theta\right)\right)}}, y\right. \\
\left.\sqrt{\frac{\alpha^{2} y^{2} t^{2}-2 \alpha^{2} c^{\prime} y t \phi+\alpha^{2} c^{\prime 2}\left(\phi^{2}-\theta\right)}{\alpha y\left(\phi^{2}-\theta\right)}} e^{\tau^{\prime}} ; \alpha\right)
\end{gathered}
$$

which after setting $b^{\prime}=-\frac{b}{d}, c^{\prime}=-c d$, $\exp \left(\frac{\tau^{\prime}}{2}\right)=\frac{1}{d}$ and using the fact that $a d-b c=1$, gives

$$
\begin{aligned}
& {[T(g) f](x, y, t ; \alpha)} \\
& \quad=\left(\frac{\alpha y}{\eta^{2}-d^{2} \theta}\right)^{\mu} f\left(\frac{\sqrt{\alpha y}(\psi \eta-c d \theta)}{\sqrt{\psi^{2}-c^{2} \theta} \sqrt{\eta^{2}-d^{2} \theta}}, y, \sqrt{\frac{\alpha\left(\psi^{2}-c^{2} \theta\right)}{y\left(\eta^{2}-d^{2} \theta\right)}} ; \alpha\right),
\end{aligned}
$$

where $\psi:=c x+$ ayt and $\eta:=d x+b y t$.

In the next section, we derive generating relations by assigning particular values to the constants $b^{\prime}$ and $c^{\prime}$ in the transformed function (3.12). Further 
we use multiplier representation (3.14) and determine matrix elements so as to derive more generating relations.

\section{Generating Relations}

First we obtain generating relations from the operator $J^{3}$ by considering the following three cases of the transformed function $\exp \left(b^{\prime} J^{+}\right) \exp \left(c^{\prime} J^{-}\right) \times$ $\left[C_{n}^{(\mu)}(x, y ; \alpha) t^{n}\right]$.

Case 1. Taking $b^{\prime}=0$ and $c^{\prime}=1$ in equation (3.12), we obtain

$$
e^{J^{-}}\left[C_{n}^{(\mu)}(x, y ; \alpha) t^{n}\right]=\left(t^{2}-\frac{2 x t}{y}+\frac{\alpha}{y}\right)^{n / 2} C_{n}^{(\mu)}\left(\frac{(x t-\alpha)}{\sqrt{t^{2}-\frac{2 x t}{y}+\frac{\alpha}{y}}}, y ; \alpha\right)
$$

Now expanding this function, we get

$$
\begin{array}{r}
\left(t^{2}-\frac{2 x t}{y}+\frac{\alpha}{y}\right)^{n / 2} C_{n}^{(\mu)}\left(\frac{(x t-\alpha)}{\sqrt{t^{2}-\frac{2 x t}{y}+\frac{\alpha}{y}}}, y ; \alpha\right) \\
=\sum_{\nu=0}^{n} \frac{(1-2 \mu-n)_{\nu}}{\nu !} C_{n-\nu}^{(\mu)}(x, y ; \alpha) t^{n-\nu}
\end{array}
$$

and further taking $t^{-1}=l$, we get

$$
\begin{array}{r}
\left(1-\frac{2 x l}{y}+\frac{\alpha l^{2}}{y}\right)^{n / 2} C_{n}^{(\mu)}\left(\frac{(x-\alpha l)}{\sqrt{1-\frac{2 x l}{y}+\frac{\alpha l^{2}}{y}}}, y ; \alpha\right) \\
=\sum_{\nu=0}^{n} \frac{(1-2 \mu-n)_{\nu}}{\nu !} C_{n-\nu}^{(\mu)}(x, y ; \alpha) l^{\nu} .
\end{array}
$$

Case 2. Taking $b^{\prime}=1$ and $c^{\prime}=0$ in equation (3.12), we obtain

$$
\begin{aligned}
& e^{J^{+}}\left[C_{n}^{(\mu)}(x, y ; \alpha) t^{n}\right] \\
& \quad=(\alpha)^{\mu+n / 2}\left(y t^{2}-2 x t+\alpha\right)^{-(\mu+n / 2)} C_{n}^{(\mu)}\left(\frac{\sqrt{\alpha}(x-y t)}{\sqrt{y t^{2}-2 x t+\alpha}}, y ; \alpha\right) t^{n},
\end{aligned}
$$

and further expanding this function, we get

$$
\begin{array}{r}
(\alpha)^{\mu+n / 2}\left(y t^{2}-2 x t+\alpha\right)^{-(\mu+n / 2)} C_{n}^{(\mu)}\left(\frac{\sqrt{\alpha}(x-y t)}{\sqrt{y t^{2}-2 x t+\alpha}}, y ; \alpha\right) \\
=\sum_{\nu=0}^{\infty}\left(\begin{array}{c}
n+\nu \\
\nu
\end{array}\right) C_{n+\nu}^{(\mu)}(x, y ; \alpha) t^{\nu} .
\end{array}
$$


Case 3. Taking $b^{\prime} c^{\prime} \neq 0, b^{\prime}=1$ and $c^{\prime}=-1$ in equation (3.12), we obtain

$$
\begin{aligned}
e^{J^{+}} e^{-J^{-}} & {\left[C_{n}^{(\mu)}(x, y ; \alpha) t^{n}\right] } \\
& =\alpha^{\mu+n / 2} y^{-n / 2}\left(y t^{2}-2 x t+\alpha\right)^{-(\mu+n / 2)} C_{n}^{(\mu)}\left(\frac{\sqrt{y}(\alpha-x t)}{\sqrt{y t^{2}-2 x t+\alpha}}, y ; \alpha\right),
\end{aligned}
$$

and further expanding this function, we get

$$
\begin{aligned}
\alpha^{\mu+n / 2} y^{-n / 2}\left(y t^{2}-2 x t+\alpha\right)^{-(\mu+n / 2)} C_{n}^{(\mu)} & \left(\frac{\sqrt{y}(\alpha-x t)}{\sqrt{y t^{2}-2 x t+\alpha}}, y ; \alpha\right) \\
& =\sum_{\nu=0}^{\infty} \frac{(2 \mu+\nu)_{n}}{n !} C_{\nu}^{(\mu)}(x, y ; \alpha) t^{\nu} .
\end{aligned}
$$

Next, we consider the case where $f(x, y, t ; \alpha)$ is a common eigenfunction of $\mathcal{C}$ and $J^{3}$, i.e., let $f(x, y, t ; \alpha)$ be a solution of the simultaneous equations

$$
\begin{aligned}
\mathcal{C} f(x, y, t ; \alpha) & =\mu(\mu-1) f(x, y, t ; \alpha), \\
J^{3} f(x, y, t ; \alpha) & =(n+\mu) f(x, y, t ; \alpha)
\end{aligned}
$$

which yield $f(x, y, t ; \alpha)=C_{n}^{(\mu)}(x, y ; \alpha) t^{n}$.

Thus, from equation (3.14) we have

$$
\begin{aligned}
{[T(g) f](x, y, t ; \alpha)=\alpha^{\mu+n / 2} y^{\mu-n / 2} } & \left(\psi^{2}-c^{2} \theta\right)^{n / 2}\left(\eta^{2}-d^{2} \theta\right)^{-(\mu+n / 2)} \\
& \times C_{n}^{(\mu)}\left(\frac{\sqrt{\alpha y}(\psi \eta-c d \theta)}{\sqrt{\psi^{2}-c^{2} \theta} \sqrt{\eta^{2}-d^{2} \theta}}, y ; \alpha\right),
\end{aligned}
$$

satisfying the relation

$$
\mathcal{C}[T(g(f)](x, y, t ; \alpha)=\mu(\mu-1)[T(g) f](x, y, t ; \alpha) .
$$

If $n$ is not an integer, then equation (4.5) has an expansion of the form

$$
[T(g) f](x, y, t ; \alpha)=\sum_{\nu=-\infty}^{\infty} j_{\nu}(g) C_{n+\nu}^{(\mu)}(x, y ; \alpha) t^{n+\nu}
$$

which simplifies to the identity

$$
\begin{aligned}
\alpha^{\mu+n / 2} y^{\mu-n / 2}\left(\psi^{2}-c^{2} \theta\right)^{n / 2}\left(\eta^{2}-d^{2} \theta\right)^{-(\mu+n / 2)} & C_{n}^{(\mu)}\left(\frac{\sqrt{\alpha y}(\psi \eta-c d \theta)}{\sqrt{\psi^{2}-c^{2} \theta} \sqrt{\eta^{2}-d^{2} \theta}}, y ; \alpha\right) \\
= & \sum_{\nu=-\infty}^{\infty} j_{\nu}(g) C_{n+\nu}^{(\mu)}(x, y ; \alpha) t^{n+\nu} .
\end{aligned}
$$

To determine $j_{\nu}(g)$, we set $x=y=\alpha=1$ in equation (4.7) and thus have

$$
j_{\nu}(g)=\left(\begin{array}{c}
n+\nu \\
n
\end{array}\right) a^{n}(-b)^{\nu} d^{-(2 \mu+n+\nu)}{ }_{2} F_{1}\left[2 \mu+n+\nu,-n ; 1+\nu ; \frac{b c}{a d}\right] \text {. }
$$


Substituting the latter expression into equation (4.7) and simplifying, we obtain the generating relation

$$
\begin{aligned}
\alpha^{\mu+n / 2} y^{\mu-n / 2}\left(\psi^{2}-c^{2} \theta\right)^{n / 2}\left(\eta^{2}-d^{2} \theta\right)^{-(\mu+n / 2)} C_{n}^{(\mu)}\left(\frac{\sqrt{\alpha y}(\psi \eta-c d \theta)}{\sqrt{\psi^{2}-c^{2} \theta} \sqrt{\eta^{2}-d^{2} \theta}}, y ; \alpha\right) \\
=\sum_{\nu=-\infty}^{\infty}\left(\begin{array}{c}
n+\nu \\
n
\end{array}\right) a^{n}(-b)^{\nu} d^{-(2 \mu+n+\nu)} \\
\quad \times{ }_{2} F_{1}\left[2 \mu+n+\nu,-n ; 1+\nu ; \frac{b c}{a d}\right] C_{n+\nu}^{(\mu)}(x, y ; \alpha) t^{n+\nu}, \quad \text { (4.9) }
\end{aligned}
$$

where $-\pi<\arg (a), \arg (d)<\pi ;\left|\frac{b c}{a d}\right|<1$.

Further, when $n$ is a nonnegative integer, say, $n=k$, we obtain the generating function

$$
\begin{aligned}
\alpha^{\mu+k / 2} y^{\mu-k / 2}\left(\psi^{2}-c^{2} \theta\right)^{k / 2}\left(\eta^{2}-d^{2} \theta\right)^{\mu-k / 2} C_{k}^{(\mu)}\left(\frac{\sqrt{\alpha y}(\psi \eta-c d \theta)}{\sqrt{\psi^{2}-c^{2} \theta} \sqrt{\eta^{2}-d^{2} \theta}}, y ; \alpha\right) \\
=\sum_{\nu=0}^{\infty}\left(\begin{array}{l}
\nu \\
k
\end{array}\right)(-1)^{\nu} a^{k} b^{\nu-k} d^{-(2 \mu+\nu)} \\
\times{ }_{2} F_{1}\left[2 \mu+\nu,-k ; \nu-k+1 ; \frac{b c}{a d}\right] C_{\nu}^{(\mu)}(x, y ; \alpha) t^{\nu} .
\end{aligned}
$$

In the next section, we obtain some results as applications of the generating relations (4.1), (4.2), (4.3), (4.9) and (4.10) which appear to be new. We also mention some interesting known applications.

\section{Applications}

We consider some applications of the generating relations obtained in the preceding section.

I. Taking $a=d=t=1$ and $c=0$ in the generating relation (4.9), we obtain

$$
\begin{aligned}
(\alpha)^{\mu+n / 2}\left(\alpha+2 b x+b^{2} y\right)^{-(\mu+n / 2)} C_{n}^{(\mu)} & \left(\frac{\sqrt{\alpha}(x+b y)}{\sqrt{\alpha+2 b x+b^{2} y}}, y ; \alpha\right) \\
& =\sum_{\nu=0}^{\infty}\left(\begin{array}{c}
n+\nu \\
n
\end{array}\right)(-b)^{\nu} C_{n+\nu}^{(\mu)}(x, y ; \alpha),
\end{aligned}
$$

which for $\alpha=y=1$ and $b=-t$ reduces to ([13]; p. 280(23))

$$
\left(1-2 x t+t^{2}\right)^{-(\mu+n / 2)} C_{n}^{(\mu)}\left(\frac{(x-t)}{\sqrt{1-2 x t+t^{2}}}\right)=\sum_{\nu=0}^{\infty}\left(\begin{array}{c}
n+\nu \\
n
\end{array}\right) C_{n+\nu}^{(\mu)}(x) t^{\nu} .
$$

Similarly, taking $a=d=t=1$ and $c=0$, we obtain the result corresponding to the generating relation (4.10). 
II. Taking $a=d=t=1$ and $b=0$ in the generating relation (4.9), we get

$$
\begin{gathered}
y^{\mu}\left(y+2 c x+c^{2} \alpha\right)^{n / 2} C_{n}^{(\mu)}\left(\frac{\sqrt{y}(x+c \alpha)}{\sqrt{y+2 c x+c^{2} \alpha}}, y ; \alpha\right) \\
=\sum_{\nu=0}^{\infty} \frac{(1-\mu-n)_{\nu}}{\nu !} c^{\nu} C_{n-\nu}^{(\mu)}(x, y ; \alpha),
\end{gathered}
$$

which for $\alpha=y=1$ and $b=-t$ reduces to

$$
\left(1-2 x t+t^{2}\right)^{n / 2} C_{n}^{(\mu)}\left(\frac{(x-t)}{\sqrt{1-2 x t+t^{2}}}\right)=\sum_{\nu=0}^{\infty} \frac{(1-\mu-n)_{\nu}}{\nu !} c^{\nu} C_{n-\nu}^{(\mu)}(x) .
$$

Similarly, taking $a=d=t=1$ and $b=0$, we obtain the results corresponding to the generating relation (4.10).

III. Taking $\mu=1$ in the generating relation (4.9), we get

$$
\begin{aligned}
\alpha^{1+n / 2} y^{1-n / 2}\left(\psi^{2}-c^{2} \theta\right)^{n / 2}\left(\eta^{2}-d^{2} \theta\right)^{-(1+n / 2)} U_{n}\left(\frac{\sqrt{\alpha y}(\psi \eta-c d \theta)}{\sqrt{\psi^{2}-c^{2} \theta} \sqrt{\eta^{2}-d^{2} \theta}}, y ; \alpha\right) \\
=\sum_{\nu=-\infty}^{\infty}\left(\begin{array}{c}
n+\nu \\
n
\end{array}\right) a^{n}(-b)^{\nu} d^{-(2+n+\nu)} \\
\quad \times{ }_{2} F_{1}\left[2+n+\nu,-n ; 1+\nu ; \frac{b c}{a d}\right] U_{n+\nu}(x, y ; \alpha) t^{n+\nu}, \quad \text { (5.4) }
\end{aligned}
$$

where $U_{n}(x, y ; \alpha)$ is given by equation (2.4).

Similarly, taking $\mu=1$, we obtain the results corresponding to the generating relations (4.1), (4.2), (4.3) and (4.10).

IV. Taking $\mu=1 / 2$ in the generating relation (4.9), we get

$$
\begin{aligned}
\alpha^{(1+n) / 2} y^{(1-n) / 2}\left(\psi^{2}-c^{2} \theta\right)^{n / 2}\left(\eta^{2}-d^{2} \theta\right)^{-(1+n) / 2} P_{n}\left(\frac{\sqrt{\alpha y}(\psi \eta-c d \theta)}{\sqrt{\psi^{2}-c^{2} \theta} \sqrt{\eta^{2}-d^{2} \theta}}, y ; \alpha\right) \\
=\sum_{\nu=-\infty}^{\infty}\left(\begin{array}{c}
n+\nu \\
n
\end{array}\right) a^{n}(-b)^{\nu} d^{-(1+n+\nu)} \\
\quad \times{ }_{2} F_{1}\left[1+n+\nu,-n ; 1+\nu ; \frac{b c}{a d}\right] P_{n+\nu}(x, y ; \alpha) t^{n+\nu}, \quad \text { (5.5) }
\end{aligned}
$$

where $P_{n}(x, y ; \alpha)$ is given by equation (2.6).

Similarly, taking $\mu=1 / 2$, we obtain the results corresponding to the generating relations (4.1), (4.2), (4.3) and (4.10).

V. Taking $\alpha=y=1$ in the generating relation (4.9), we obtain

$$
\left(c^{2}+a^{2} t^{2}+2 a c x t\right)^{n / 2}\left(d^{2}+b^{2} t^{2}+2 b d x t\right)^{-(\mu+n / 2)}
$$




$$
\begin{aligned}
\times C_{n}^{(\mu)} & \left(\frac{\left(c d+a b t^{2}+(a d+b c) x t\right)}{\sqrt{c^{2}+a^{2} t^{2}+2 a c x t} \sqrt{d^{2}+b^{2} t^{2}+2 b d x t}}\right) \\
= & \sum_{\nu=-\infty}^{\infty}\left(\begin{array}{c}
n+\nu \\
n
\end{array}\right) a^{n}(-b)^{\nu} d^{-(2 \mu+n+\nu)} \\
& \times{ }_{2} F_{1}\left[2 \mu+n+\nu,-n ; 1+\nu ; \frac{b c}{a d}\right] C_{n+\nu}^{(\mu)}(x) t^{n+\nu},
\end{aligned}
$$

where $C_{n}^{(\mu)}(x)$ is given by equation (1.6).

Similarly, taking $\alpha=y=1$, we obtain the result corresponding to the generating relation (4.10). The generating relations $(4.1)-(4.3)$ reduce to ([9]; pp. $52-$ $53(6-8))$.

VI. Taking $\alpha=y=\mu=1$ in the generating relation (4.9), we obtain

$$
\begin{aligned}
&\left(c^{2}+a^{2} t^{2}+2 a c x t\right)^{n / 2}\left(d^{2}+b^{2} t^{2}+2 b d x t\right)^{-(1+n / 2)} \\
& \times U_{n}\left(\frac{\left(c d+a b t^{2}+(a d+b c) x t\right)}{\sqrt{c^{2}+a^{2} t^{2}+2 a c x t} \sqrt{d^{2}+b^{2} t^{2}+2 b d x t}}\right) \\
&=\sum_{\nu=-\infty}^{\infty}\left(\begin{array}{c}
n+\nu \\
n
\end{array}\right) a^{n}(-b)^{\nu} d^{-(2+n+\nu)} \\
& \quad \times{ }_{2} F_{1}\left[2+n+\nu,-n ; 1+\nu ; \frac{b c}{a d}\right] U_{n+\nu}(x) t^{n+\nu},
\end{aligned}
$$

where $U_{n}(x)$ is given by equation (1.2).

Similarly, taking $\alpha=y=\mu=1$, we obtain the results corresponding to the generating relations (4.1), (4.2), (4.3) and (4.10).

VII. Taking $\alpha=y=1$ and $\mu=1 / 2$ in the generating relation (4.9), we obtain

$$
\begin{aligned}
\left(c^{2}+a^{2} t^{2}+2 a c x t\right)^{n / 2} & \left(d^{2}+b^{2} t^{2}+2 b d x t\right)^{-(1+n) / 2} \\
\times P_{n}( & \left.\frac{\left(c d+a b t^{2}+(a d+b c) x t\right)}{\sqrt{c^{2}+a^{2} t^{2}+2 a c x t} \sqrt{d^{2}+b^{2} t^{2}+2 b d x t}}\right) \\
= & \sum_{\nu=-\infty}^{\infty}\left(\begin{array}{c}
n+\nu \\
n
\end{array}\right) a^{n}(-b)^{\nu} d^{-(1+n+\nu)} \\
& \quad \times{ }_{2} F_{1}\left[1+n+\nu,-n ; 1+\nu ; \frac{b c}{a d}\right] P_{n+\nu}(x) t^{n+\nu},
\end{aligned}
$$

where $P_{n}(x)$ is given by equation (2.14). For $a=d=t=1, b=-t$ and $c=0$, equation (5.8) reduces to ([13]; p. 169(7))

$$
\left(1-2 x t+t^{2}\right)^{-(1+n) / 2} P_{n}\left(\frac{(x-t)}{\sqrt{1-2 x t+t^{2}}}\right)=\sum_{\nu=-\infty}^{\infty}\left(\begin{array}{c}
n+\nu \\
n
\end{array}\right) P_{n+\nu}(x) t^{\nu} .
$$


Similarly, taking $\alpha=y=1$ and $\mu=1 / 2$, we obtain the results corresponding to the generating relations (4.1), (4.2), (4.3) and (4.10).

It is interesting to note that we can express the results involving 2V1PGP $C_{n}^{(\mu)}(x, y ; \alpha)$ in terms of GHP $P_{n}(2, x, y,-\mu, \alpha)$ by using equation $(2.7)$.

In the next section, we will discuss possible future directions of the present work.

\section{Concluding Remarks}

In this paper we have derived the generating relations involving 2V1PGP

$C_{n}^{(\mu)}(x, y ; \alpha)$ by using the representation theory of the Lie group SL(2). Also, we have obtained many new results for the polynomials associated with 2V1PGP.

Further, note that the generating relations obtained in this paper can be used to find many interesting relations involving other polynomials. As a specific example, we consider the derivation of a generating relation involving a new family of two variable orthogonal polynomials $R_{n}(x, y)$ defined by the generating function ([2]; p. 368(28))

$$
\left(1+2(x-y) t+(x+y)^{2} t^{2}\right)^{-1 / 2}=\sum_{n=0}^{\infty} R_{n}(x, y) t^{n} .
$$

The polynomials $R_{n}(x, y)$ can be expressed in terms of Legendre polynomials $P_{n}(x)$ by the relation

$$
R_{n}(x, y)=(x+y)^{n} P_{n}\left(\frac{y-x}{x+y}\right),
$$

which implies

$$
P_{n}(x)=R_{n}\left(\frac{1-x}{2}, \frac{1+x}{2}\right) .
$$

We can infer from equations (1.8) and (6.1) that

$$
C_{n}^{(1 / 2)}\left((y-x),(x+y)^{2} ; 1\right)=R_{n}(x, y) .
$$

Now, taking $\mu=\frac{1}{2}, \alpha=1$. Replacing $x$ by $(y-x)$ and $y$ by $(x+y)^{2}$ in the generating relation (4.9) and using equation (6.4), we obtain

$$
\begin{aligned}
& Y^{-n / 2}\left(a^{2} t^{2} Y+2 a c t X\right.\left.+c^{2}\right)^{n / 2}\left(b^{2} t^{2} Y+2 b d t X+d^{2}\right)^{-(1+n) / 2} \\
& \times P_{n}\left(\frac{\sqrt{Y}\left((a d t+b c t) X+a b t^{2} Y+c d\right)}{\sqrt{a^{2} t^{2} Y+2 a c t X+c^{2}} \sqrt{b^{2} t^{2} Y+2 b d t X+d^{2}}}, Y\right) \\
&=\sum_{\nu=-\infty}^{\infty}\left(\begin{array}{c}
n+\nu \\
n
\end{array}\right) a^{n}(-b)^{\nu}(d)^{-(1+n+\nu)} \\
& \quad \times{ }_{2} F_{1}\left[1+n+\nu,-n ; 1+\nu ; \frac{b c}{a d}\right] R_{n+\nu}(x, y) t^{n+\nu},
\end{aligned}
$$


where $X:=(y-x) ; Y:=(x+y)^{2} ;-\pi<\arg (a), \arg (d)<\pi ;\left|\frac{b c}{a d}\right|<1$, and $P_{n}(x, y)$ denotes two variable Legendre polynomials (obtained by taking $\alpha=1$ in equation (2.6)).

By the same procedure we can obtain the results corresponding to the generating relations (4.1), (4.2), (4.3), (4.10), (5.1) and (5.3).

Similarly, we can derive generating relations involving two variable polynomials $S_{n}(x, y)$ defined by the generating function ([2]; p. 368(24))

$$
\left(1-2 y t+\left(y^{2}-4 x\right) t^{2}\right)^{-1 / 2}=\sum_{n=0}^{\infty} S_{n}(x, y) t^{n}
$$

which on account of the generating function (2.14) of Legendre polynomials $P_{n}(x)$, suggests that

$$
S_{n}(x, y)=\left(y^{2}-4 x\right)^{n / 2} P_{n}\left(\frac{y}{\sqrt{y^{2}-4 x}}\right) ;
$$

also, we note that

$$
P_{n}(x)=S_{n}\left(-\frac{1}{4}\left(1-x^{2}\right), x\right) .
$$

Now equations (1.8) and (6.6) allow us to conclude that

$$
C_{n}^{(1 / 2)}\left(\frac{y^{3 / 2}}{\sqrt{y^{2}-4 x}}, y ; 1\right)=\left(y-\frac{4 x}{y}\right)^{-n / 2} S_{n}(x, y),
$$

which can be used to derive generating relations involving $S_{n}(x, y)$.

It is also worth noting that

$$
C_{n}^{(\mu)}(x, y ; \alpha)=\frac{1}{\alpha^{\mu}}\left(\frac{y}{\alpha}\right)^{n / 2} C_{n}^{(\mu)}\left(\frac{x}{\sqrt{\alpha y}}\right),
$$

from which it follows that

$$
C_{n}^{(\mu)}(x, y ; \alpha)=\frac{(2 \mu)_{n}}{\left(\mu+\frac{1}{2}\right)_{n} \alpha^{\mu}}\left(\frac{y}{\alpha}\right)^{n / 2} P_{n}^{\left(\mu-\frac{1}{2}, \mu-\frac{1}{2}\right)}\left(\frac{x}{\sqrt{\alpha y}}\right),
$$

where $P_{n}^{(\alpha, \alpha)}(x)$ denotes ultraspherical polynomials [13].

Expressions (6.10) and (6.11) can be used to transform the results obtained for the 2V1PGP $C_{n}^{(\mu)}(x, y ; \alpha)$ to the results for the GP $C_{n}^{(\mu)}(x)$ or vice-versa.

The theory of special functions of mathematical physics is known to be deeply rooted in the theory of Lie algebras and groups. In specific irreducible representations, these functions appear as matrix elements of group operators, and also as basis vectors of representation spaces. This interpretation provides a vantage standpoint in the study of the properties of special functions, since generating relations, orthogonality properties and addition formulas are naturally obtained within this algebraic setting [10]-[12], [14].

It appears now clear that the so-called basic or $q$-special functions [6] are similarly related to the representation theory of quantum algebras and quantum 
groups. The matrix elements of certain algebra generators in irreducible representations are in fact expressible in terms of $q$-hypergeometric series (see, e.g., [5] and the references therein). Taking the quantum algebra $\mathcal{U}_{q}(\operatorname{su}(1,1))$ as an example, the authors of [5] show that the metaplectic representation of the real form $\mathcal{U}_{q}(\mathrm{su}(1,1))$ of the quantum algebra $\mathcal{U}_{q}(\mathrm{sl}(2))$ provides a group-theoretic setting for certain basic orthogonal polynomials generalizing usual Gegenbauer polynomials.

In a forthcoming investigation, we will consider the possibility of $q$-generalization of Gegenbauer polynomials. We will also study the connection of $q$ generalized Gegenbauer polynomials with quantum algebras.

Moreover, the study of other forms of Gegenbauer and $q$-Gegenbauer polynomials from the standpoint of both applications and their connections with various Lie algebras and quantum algebras is also an interesting problem for further research.

\section{ACKNOWLEDGMENT}

The authors are thankful to the referee for valuable suggestions on improving the presentation of the paper.

\section{REFERENCES}

1. G. Dattoli, S. Lorenzutta, and C. Cesarano, From Hermite to Humbert polynomials. Rend. Istit. Mat. Univ. Trieste 35(2003), No. 1-2, 37-48 (2004).

2. G. Dattoli, P. E. Ricci, and C. Cesarano, A note on Legendre polynomials. Int. J. Nonlinear Sci. Numer. Simul. 2(2001), No. 4, 365-370.

3. G. Dattoli, P. E. Ricci, and C. Cesarano, A note on multi-index polynomials of Dickson type and their applications in quantum optics. J. Comput. Appl. Math. 145(2002), No. 2, 417-424.

4. P. J. DAVIS, Interpolation and approximation. Republication, with minor corrections, of the 1963 original, with a new preface and bibliography. Dover Publications, Inc., New York, 1975.

5. R. Floreanini and L. Vinet, Quantum algebra approach to $q$-Gegenbauer polynomials. Proceedings of the Fourth International Symposium on Orthogonal Polynomials and their Applications (Evian-Les-Bains, 1992). J. Comput. Appl. Math. 57(1995), No. 1-2, 123133.

6. G. Gasper and M. Rahman, Basic hypergeometric series. Encyclopedia of Mathematics and its Applications, 35. Cambridge University Press, Cambridge, 1990.

7. H. W. Gould, Inverse series relations and other expansions involving Humbert polynomials. Duke Math. J. 32(1965), 697-711.

8. S. Khan and M. A. Pathan, Lie-theoretic generating relations of Hermite 2D polynomials. Proceedings of the International Conference on Special Functions and their Applications (Chennai, 2002). J. Comput. Appl. Math. 160(2003), No. 1-2, 139-146.

9. E. B. McBride, Obtaining generating functions. Springer Tracts in Natural Philosophy, Vol. 21. Springer-Verlag, New York-Heidelberg, 1971.

10. W. Miller, Jr., Lie theory and special functions. Mathematics in Science and Engineering, Vol. 43. Academic Press, New York-London, 1968. 
11. A. F. Nikiforov, S. K. Suslov, and V. B. Uvarov, Classical orthogonal polynomials of a discrete variable. (Translated from the Russian) Springer Series in Computational Physics. Springer-Verlag, Berlin, 1991.

12. A. F. Nikiforov and V. B. Uvarov, Special functions of mathematical physics. A unified introduction with applications. (Translated from the Russian) Birkhäuser Verlag, Basel, 1988.

13. E. D. Rainville, Special functions. The Macmillan Co., New York, 1960.

14. N. JA. Vilenkin, Special functions and the theory of group representations. (Translated from the Russian) Translations of Mathematical Monographs, Vol. 22. American Mathematical Society, Providence, R. I., 1968.

15. L. Weisner, Group-theoretic origin of certain generating functions. Pacific J. Math. 5(1955), 1033-1039.

(Received 15.03.2005)

Authors' addresses:

S. Khan and A. Mittal

Department of Mathematics

Aligarh Muslim University, Aligarh-202002

India

E-mail: subuhi@lycos.com

G. Yasmin

Women's College, Aligarh Muslim University

Aligarh-202002

India 